\title{
LOS FACTORES DE DIFERENCIACIÓN SOCIOESPACIAL DE LA POBLACIÓN EN EL MUNICIPIO DE MARBELLAA LA LUZ DEL CENSO DE 2001
}

\author{
JUAN JOSÉ NATERA RIVAS
}

\begin{abstract}
RESUMEN
A partir de la información del Censo de Población de 2001 a nivel de sección censal, en el presente estudio nos interesamos por los factores que subyacen en la diferenciación socioespacial de la población en el municipio de Marbella. Además, comparamos los resultados con los obtenidos en otro estudio utilizando información referida a 1991, para lo cual se han seleccionado las mismas variables, la misma referencia espacial y el mismo método de análisis de la información.
\end{abstract}

\section{ABSTRACT}

Using the data from 2001 Population Census provided at census tract level, this paper focuses on the factors underlying the sociospatial differentiation of the inhabitants of Marbella. Along with this, we compare the results with those obtained before using information referred to 1991, using the same variables, spatial references and methods in order to maintain comparability.

\section{INTRODUCCIÓN}

En los 10 años que van desde el Censo de Población y Viviendas de 1991 y el realizado en 2001, el municipio de Marbella ha visto cómo el número de residentes censados en él se ha elevado en más de 10.000 personas, hasta alcanzar los 99.991 censados en la última fecha, y este aumento ha sido común a los cuatro distritos en los que se divide el municipio: el primero, que engloba el núcleo de la ciudad de Marbella (figura 1 a), el segundo, que hace lo propio con el correspondiente a la barriada de San Pedro Alcántara (figura 1 b), el tercero, situado entre ambos, y el cuarto, que se extiende desde Arroyo Primero hacia el límite oriental del municipio (figura $1 \mathrm{c}$ ). Volviendo al aumento de 
población experimentado por estas unidades espaciales, es cierto que en todas se ha producido, pero de forma variable: son los distritos 3 y 4 los que, en términos porcentuales, mayor incremento de población han sufrido, $60,72 \%$ y $61,1 \%$ respectivamente, a gran distancia del segundo $(29,54 \%)$ y del primero $(12,72 \%)$. Y aunque es cierto que ha sido en este último distrito en el que el incremento de la población ha sido mayor en números absolutos, las diferencias con respecto al resto no son excesivamente marcadas (cuadro 1).

Desde el punto de vista de las características estructurales, el conjunto de la población censada en Marbella ha experimentado durante el último intercenso un envejecimiento tanto por la base como por la cúspide. Así, al mismo tiempo que los menores de 15 años veían cómo el porcentaje que representan sobre el total de la población ha caído al $17 \%$, frente a poco menos del $25 \%$ en 1991, la población de 65 años o más ha experimentado la situación opuesta, resultando que en el último momento censal uno de cada 10 marbellíes integraba el grupo de los ancianos; y, una vez más, este proceso puede extrapolarse a la totalidad de los distritos censales (cuadro 1).

Por otro lado, el número de hogares en los que se agrupa la población municipal se ha incrementado de forma muy apreciable, hasta casi doblarse, resultando ser el aumento considerablemente más marcado en los distritos 3 y 4 , tal y como se aprecia en el cuadro 1 . De cualquier forma, y como refle-

\section{FIGURA 1 A \\ DIVISIÓN CENSAL DE LA CIUDAD DE MARBELLA (1991)}

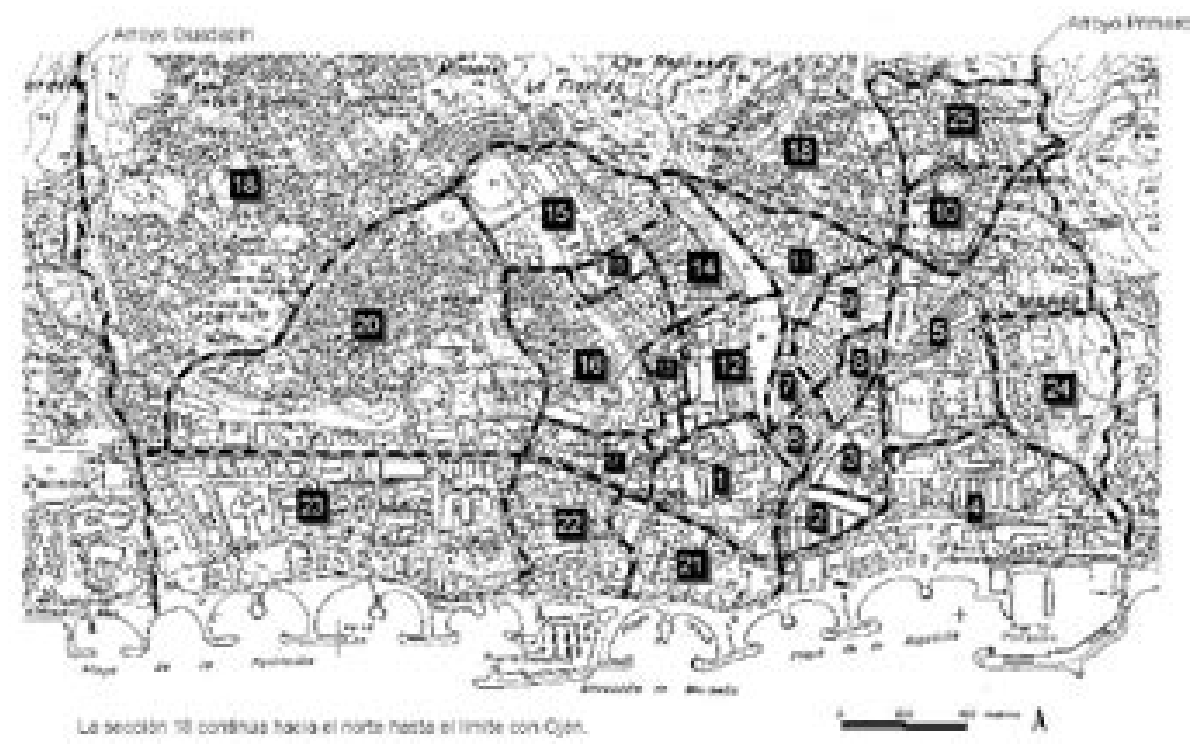




\section{FIGURA 1 B}

DIVISIÓN CENSAL DE SAN PEDRO ALCÁNTARA (1991)

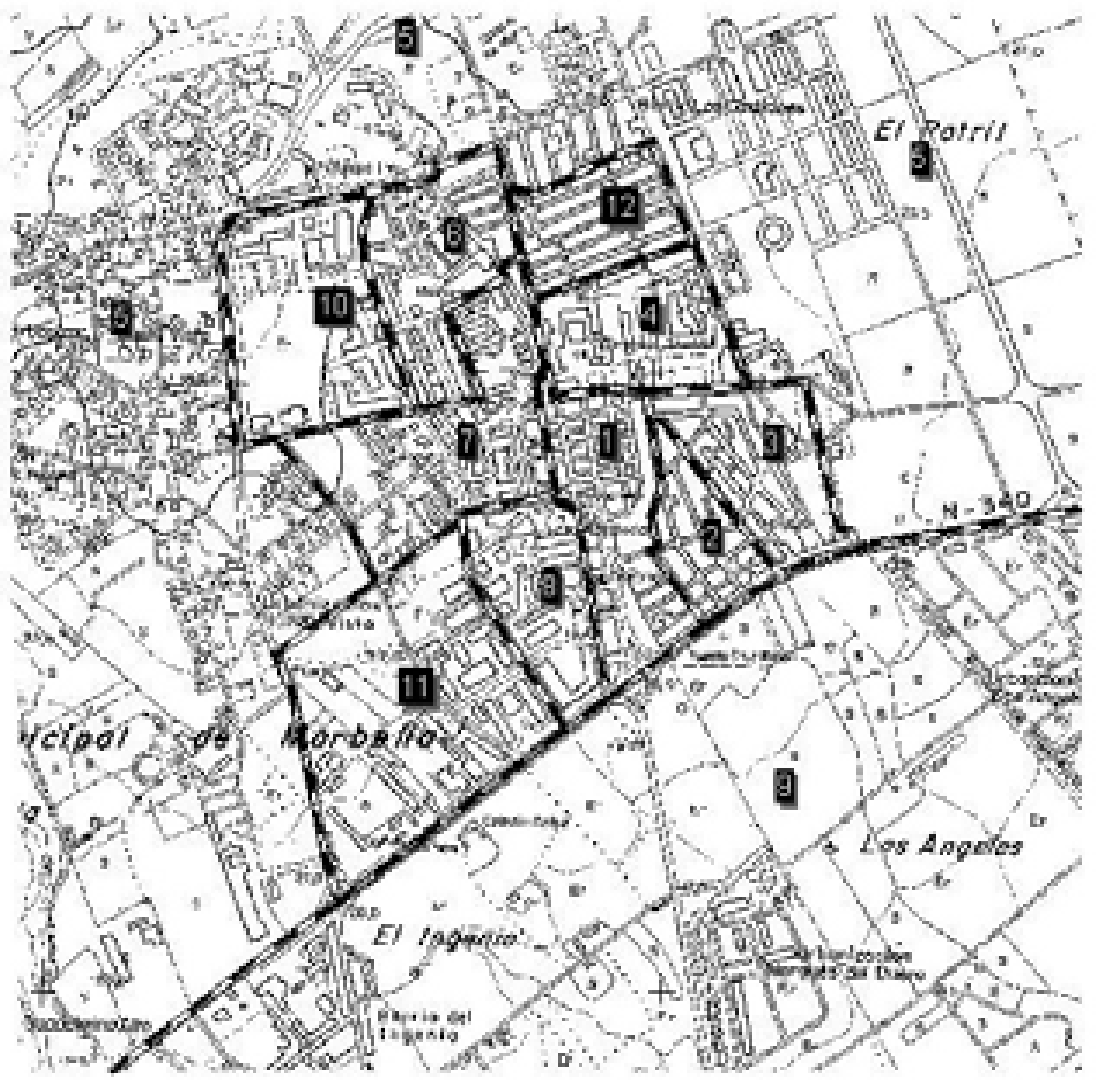

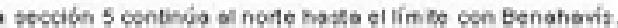

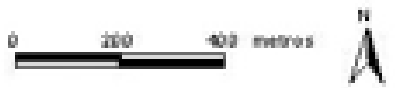




\section{FIGURA 1 C LOCALIZACIÓN Y SECCIONADO DE LOS DISTRITOS 3 Y 4 (1991)}

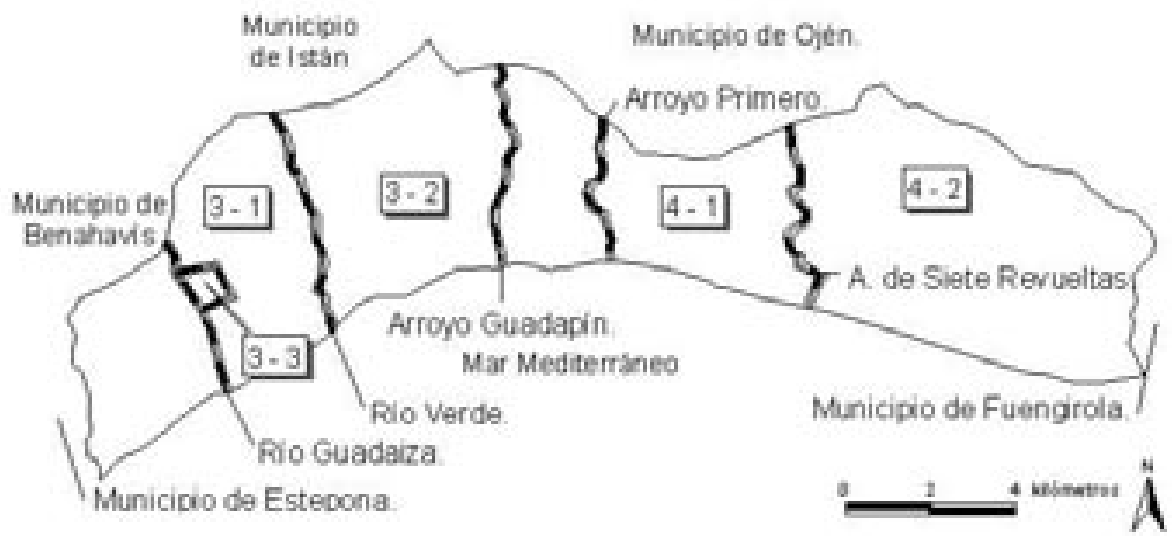

jo del ritmo desenfrenado de construcción que ha sufrido el municipio, este importante aumento de hogares queda empequeñecido al compararlo con el correspondiente a las viviendas; así, según el Nomenclátor de 1991, en todo el municipio había un total de 49.184 viviendas, mientras que el Censo de 2001 indicaba que ya eran 80.172 , de las cuales tan sólo 35.252 principales y 44.920 secundarias, !una cifra similar al total que existía tan sólo diez años antesi

\section{CUADRO 1}

\section{VALORES DE VARIABLES SELECCIONADAS EN LOS CENSOS DE} 1991 Y 2001

\begin{tabular}{|l|c|c|c|c|c|c|c|c|c|c|}
\hline & \multicolumn{2}{|c|}{ Total } & \multicolumn{2}{c|}{ Distrito 1 } & \multicolumn{2}{c|}{ Distrito 2 } & \multicolumn{2}{c|}{ Distrito 3 } & \multicolumn{2}{c|}{ Distrito 4 } \\
\hline Censo & 1991 & 2001 & 1991 & 2001 & 1991 & 2001 & 1991 & 2001 & 1991 & 2001 \\
\hline Total Población & 80.599 & 99.991 & 49.232 & 55.530 & 19.158 & 24.818 & 6.900 & 11.090 & 5.309 & 8.553 \\
\hline \% > de 15 años & 23,89 & 17,41 & 23,74 & 16,89 & 25,31 & 17,15 & 23,12 & 18,91 & 21,21 & 19,59 \\
\hline \% de 64 años & 8,37 & 10,02 & 8,09 & 10,44 & 7,7 & 10,23 & 7,18 & 8,52 & 14,56 & 8,64 \\
\hline $\mathrm{N}^{\circ}$ hogares & 19.574 & 35.252 & 11.058 & 19.262 & 5.243 & 8.096 & 1.968 & 4.660 & 1.305 & 3.234 \\
\hline \% ocupados & 42,29 & 53,27 & 43,04 & 53,35 & 41,69 & 51,74 & 45,8 & 55,17 & 33,09 & 54,82 \\
\hline \% parados & 13,71 & 8,72 & 14,41 & 8,55 & 13,85 & 8,66 & 11,16 & 9,08 & 10,21 & 9,49 \\
\hline \% jubilados & 6,45 & 10,31 & 6,22 & 9,16 & 6,82 & 9,36 & 4,84 & 9,75 & 9,31 & 10,76 \\
\hline \% con primaria & 57,82 & 40,71 & 56,73 & 40,52 & 65,73 & 45,97 & 54,39 & 33,23 & 44,12 & 36,2 \\
\hline \% est. superiores & 5,58 & 10,02 & 5,46 & 9,58 & 3,16 & 7,31 & 10,01 & 15,47 & 9,46 & 13,85 \\
\hline
\end{tabular}

También desde el punto de vista de las características de la instrucción, o de determinadas variables económicas de la población, el conjunto de los censados en el municipio ha experimentado algunos cambios en el último in- 
tercenso. A modo de ejemplo, puede indicarse que, en el momento del censo, el porcentaje sobre la población de 16 años o más de ocupados era mayor en 2001 que en 1991, o que el de parados era considerablemente menor en la primera fecha que en la segunda, al tiempo que, de forma consecuente con el aumento de la población adulta mayor, en los 10 años que median entre ambos censos se había incrementado el porcentaje de jubilados sobre la población de 16 años o más. Y desde el punto de vista de la instrucción, se ha registrado una mejora notable en los niveles de estudio de la población, desde el momento en que el porcentaje de aquellos de 10 años o más que cuentan con una credencial máxima de estudios primarios completos ha disminuido más de 17 puntos porcentuales, al tiempo que, en el otro extremo, el porcentaje de titulados superiores ha aumentado desde el 5,58\% de 1991 al 10,02\% del último Censo.

Junto a estos cambios experimentados por la población, también la trama urbana ha sufrido mutaciones. Sin ánimo de ser exhaustivos, podemos indicar que en algunas áreas se han registrado importantes procesos de renovación urbana (como en el área de el Barrio Nuevo, en la que este proceso aún continua), en otras se ha asistido a una multiplicación del espacio construido (como en el extremo oriental del casco marbellí, con su correlato en el extremo occidental del distrito 4), siendo norma general la densificación constructiva del espacio municipal. Aunque bien es cierto que no todas las viviendas levantadas están ocupadas, también lo es el que, como al comienzo indicamos, Marbella ha experimentado un aumento de población, que se ha centrado mayoritariamente en algunas secciones censales concretas. Reflejo de los importantes aumentos de población experimentados por algunas de ellas es el desgajamiento de parte de su extensión para la conformación de otra, con el objetivo de mantener los volúmenes de población dentro de los límites marcados por la legislación; es el caso, por citar algunos ejemplos, de las secciones 13 y 15, desgajadas de la antigua 5, en San Pedro, la 30 del distrito 1, proveniente de la ruptura de la 20 en Marbella, etc. Como resultado de este proceso, de las 42 secciones censales a las que quedaba referida la información a nivel intraurbano en el Censo de 1991, se ha pasado a las 60 del Censo de 2001.

Hace algunos años realizábamos un estudio ${ }^{1}$ acerca de las diferencias que la población mostraba en su distribución espacial en función de algunas variables básicas, como estructura por edad, instrucción, ocupación, origen, etc. Lo concluíamos mostrando cómo eran dos los factores que subyacían en dichas diferencias espaciales: el primero, el más importante, que fue identificado con el estatus social. A él se unía otro, identificado como el estatus familiar. Básicamente las secciones urbanas de mayor estatus se localizaban en los distritos

1. NATERA RIVAS, J. J.: Heterogeneidad social y diferenciación residencial en Marbella, Málaga 2002. 
3 y 4 y en la porción costera occidental de la ciudad de Marbella, al tiempo que los que más bajo puntuaban en esta supervariable se localizaban en el núcleo de San Pedro Alcántara, a las que se añadían algunas secciones colindantes con el centro histórico marbellí. Por su parte, eran las secciones sampedreñas las que presentaban unos ciclos familiares más jóvenes, mientras que el centro de la ciudad de Marbella se encontraba en un ciclo familiar maduro.

Como acabamos de indicar, los cambios experimentados por el conjunto de la población marbellí han sido de cierta consideración, por lo cual podemos plantearnos si los factores que subyacen en la actual diferenciación espacial de la población en función de determinadas variables, que efectivamente aparece al cartografiar la información correspondiente al Censo de 2001, son los mismos que eran en 1991, y, si esto es así, si las secciones de mayor estatus social continúan siendo las mismas, o si la distribución en el espacio de los ciclos familiares ha experimentado alguna mutación.

\section{LAS VARIABLES DE PARTIDA}

El objetivo de la presente contribución es, por un lado, indagar acerca de los factores que subyacen en la distribución espacial de la población censada en Marbella en 2001; por otro, comprobar si se han registrado cambios con respecto a la situación correspondiente a 10 años atrás. Siendo esto así, la elección tanto de las variables como la distribución espacial a la que quedarán adscritas están en función de aquellas que fueron seleccionadas al trabajar con la información de 1991, con el fin de mantener en lo posible la comparabilidad entre ambos resultados. En relación con la segunda, hemos procedido a revertir el proceso de división de las secciones censales ocurrido en los últimos 10 años, de forma que la información queda referida a las mismas 42 secciones que existían en 1991, 25 en distrito 1, 12 en el segundo, 3 en el tercero y 2 en el cuarto.

$\mathrm{Y}$ con respecto a las variables, son 16 las que hemos seleccionado para la realización del análisis. Un primer grupo son identificativas del estadio de vida familiar. El porcentaje de población menor de 15 años, junto con el correspondiente a aquella de 65 años o más; el porcentaje de personas principales del hogar menores de 35 años junto con aquellas de 55 años o más. También se ha incluido en el análisis la relación de fecundidad, calculada como el número de niños menores de 5 años por cada mujer en edad fecunda (15 y 44 años). Por último, se ha añadido el porcentaje de solteras de entre 20 y 44 años, cubriendo de esta forma el grueso de las solteras mayores de edad en edad fecunda ${ }^{2}$.

2. La población empleada en 1991 eran aquellas mujeres de entre 18 y 44 años, pero, por la manera de presentar la fuente la información en 2001 a nivel de sección, no puede 
Un segundo conjunto de variables queda referido al nivel de instrucción de los censados, y hemos seleccionado los dos extremos de la escala. Por un lado, el porcentaje de población de 10 años o más con educación superior, por otro el porcentaje de población de 20 años o más con un máximo de primaria completa. Seleccionando esta última variable se cubre tanto a la población analfabeta proveniente de las generaciones anteriores a la implantación de la educación primaria obligatoria, como a aquella otra que, por ser obligatoria, tuvo que asistir a la escuela durante la educación básica, pero que ya no continuó -ni, por el límite de edad que hemos impuesto, probablemente no continuará- estudiando ${ }^{3}$.

El tercer bloque está en relación con las características ocupacionales de la población marbellí, y está conformado por un total de seis variables. La primera de ellas se corresponde con el porcentaje de ocupados en profesiones superiores, entendiendo por tales aquellas que necesitan para su desempeño de la posesión de un título superior, o bien que implican un cargo directivo; junto a ellos se ha añadido el porcentaje de ocupados no especializados, y el porcentaje de ocupados dedicados a profesiones manuales, englobando en ellos a los empleados en la construcción, la industria manufacturera y a los operadores de maquinaria. Estos tres agrupamientos aglutinan virtualmente al mismo tipo de ocupados que fueron censados en 1991, con lo que se mantiene la comparabilidad entre ambos momentos. A estas tres se han añadido, por un lado el porcentaje de empresarios que emplean y el porcentaje de trabajadores eventuales, ambos sobre el total de ocupados; por otro el porcentaje de población de 16 años o más jubilada.

Las dos variables restantes que hemos utilizado en nuestro análisis hacen referencia al origen de la población, el porcentaje de inmigrantes extranjeros y el correspondiente a los provinciales ${ }^{4}$ sobre la población total, orígenes que,

construirse; por ello hemos tenido que emplear la población femenina de 20 años o más. Pese a ello, estimamos que la comparabilidad entre ambas variables continúa siendo elevada.

3. Al igual que ocurría con la variable "solteras", la manera en la que la fuente presenta la información a nivel de sección censal no nos permite acercarnos al nivel de instrucción de la población de 18 y 19 años, por ello esta variable no muestra una correspondencia exacta con la que empleamos con la información de 1991, que contemplaba a los censados de 18 años o más.

4. La variable porcentaje de inmigrantes provinciales sobre la población total no ha sido construida de la misma forma que en 1991. Debido al extraordinario nivel de desagregación de la información referente a 1991 del que dispusimos, fue posible desarrollar un método para evaluar la natalidad diferida y ajustar así el número de personas provenientes realmente de otros municipios de la provincia (cfr. Natera, 2002, 106-108). No obstante, esto no ha sido posible con la información de 2001, por lo que hemos considerado como inmigrantes provinciales a los nacidos en un municipio malagueño diferente al marbellí. 
tal y como ocurría en 1991, presentan importantes diferencias en su localización intramunicipal.

\section{LOS FACTORES DE DIFERENCIACIÓN EN 2001}

Por razones de espacio no podemos ofrecer de forma detallada la información referente a la distribución espacial de estas variables, por lo que tan sólo indicaremos que variables referidas a características opuestas de la población (como nivel de estudio, tipo de ocupación, origen...) presentan distribuciones espaciales diferentes. Por ello, parece razonable suponer que todavía en 2001 en la distribución de la población marbellí subyace una serie de dimensiones, para cuya identificación hemos recurrido a un análisis factorial. Como es sabido, este método trata de sintetizar la información aportada por un número elevado de variables en un número mínimo e imprescindible de nuevas variables, o factores, que concentran la información de manera sintética. El resultado del análisis, de componentes principales, y cuya matriz factorial ha sido rotada según el método varimax, se ofrece en los dos cuadros siguientes.

CUADRO 2 A

SOLUCIÓN FACTORIAL DEL MUNICIPIO DE MARBELLA (16 VARIABLES)

\begin{tabular}{|c|c|c|c|}
\hline & Valor propio & $\begin{array}{c}\text { Varianza } \\
\text { explicada }\end{array}$ & $\begin{array}{c}\text { Varianza } \\
\text { Acumulada }\end{array}$ \\
\hline Factor 1 & 7,14 & $44,6 \%$ & $44,6 \%$ \\
\hline Factor 2 & 4,84 & $30,3 \%$ & $74,9 \%$ \\
\hline Factor 3 & 1,25 & $7,8 \%$ & $82,7 \%$ \\
\hline
\end{tabular}

CUADRO 2 B.

ESTRUCTURA FACTORIAL DEL MUNICIPIO DE MARBELLA

\begin{tabular}{|l|c|c|c|c|}
\hline Variable & Comunalidad & factor 1 & factor 2 & factor 3 \\
\hline Oficios superiores & 0,96 & $-0,98$ & 0,06 & 0,03 \\
\hline Población con estudios superiores & 0,93 & $-0,96$ & 0,06 & 0,05 \\
\hline Inmigrantes extranjeros & 0,88 & $-0,86$ & 0,18 & 0,32 \\
\hline Empresarios que emplean & 0,72 & $-0,83$ & $-0,15$ & 0,09 \\
\hline Población con primaria completa & 0,89 & 0,95 & $-0,04$ & 0,02 \\
\hline Trabajadores manuales & 0,84 & 0,91 & $-0,12$ & $-0,07$ \\
\hline Trabajadores eventuales & 0,74 & 0,85 & 0,06 & 0,10 \\
\hline Trabajadores no especializados & 0,77 & 0,82 & 0,08 & 0,30 \\
\hline Inmigrantes provinciales & 0,71 & 0,72 & $-0,21$ & $-0,39$ \\
\hline
\end{tabular}




\begin{tabular}{|l|c|c|c|c|}
\hline Variable & Comunalidad & factor 1 & factor 2 & factor 3 \\
\hline Menores de 15 años & 0,86 & 0,08 & $-0,86$ & 0,34 \\
\hline 65 años o más & 0,87 & 0,07 & 0,93 & $-0,02$ \\
\hline Cabezas de familia de 55 años o más & 0,87 & 0,14 & 0,91 & $-0,15$ \\
\hline Solteras & 0,84 & $-0,19$ & 0,86 & $-0,25$ \\
\hline Jubilados & 0,79 & $-0,16$ & 0,87 & 0,02 \\
\hline Cabezas de familia menores de 35 años & 0,78 & $-0,08$ & $-0,34$ & 0,81 \\
\hline Relación de fecundidad & 0,76 & $-0,03$ & $-0,54$ & 0,68 \\
\hline
\end{tabular}

El análisis ha diferenciado un total de tres factores, que en conjunto explican una parte muy importante del total de la varianza, concretamente el $82,7 \%$, aunque el peso de cada uno de ellos es diferente: el primer factor explica por sí sólo el 44,6\% de la varianza total, frente el 30,3\% del segundo y un exiguo $7,8 \%$ del tercero. Además, y a la vista de las elevadas comunalidades que presentan las variables, su elección continua siendo correcta cuando lo que se quiere es indagar acerca de la diferenciación intraurbana de la población del municipio marbellí.

A la identificación del primer factor contribuye un total de 9 variables, todas con puntuaciones que podrían considerarse como elevadas o muy elevadas. Un primer grupo de variables, que intuitivamente podríamos relacionar estrechamente entre sí, queda compuesto por el porcentaje de población con estudios superiores completos, el porcentaje de ocupados con empleos superiores y el porcentaje de empresarios que emplean, todas con signo negativo. A ellas se añade, también con signo negativo, el porcentaje de inmigrantes de origen extranjero. En el otro extremo, el análisis ha agrupado en este factor también a una serie de variables que resultan ser el opuesto de las anteriores; puntuando en su totalidad con signo positivo, la población con un máximo de instrucción de primaria completa, los ocupados eventuales y aquellos otros con oficios manuales o no especializados, junto al porcentaje de inmigrantes provinciales componen la nómina de este segundo grupo, alcanzando todas puntuaciones elevadas, la menor correspondiente a los inmigrantes provinciales. Con esta información, la identificación del factor parece ser clara: hace referencia al estatus social, de forma que cuanto más elevadas sean las puntuaciones positivas más bajo será este y, al contrario, cuanto menores sean las negativas mayor será el estatus social de la sección censal.

Son un total de cuatro variables las que dan significado al segundo factor, tres de ellas con signo negativo: el porcentaje de población con 65 años o más, el porcentaje de personas principales del hogar de 55 años o más, y el porcentaje de solteras. La cuarta, el porcentaje de menores de 15 años, es la única que puntúa con signo positivo. Así las cosas, la relación de este factor con el estatus familiar parece probable, desde el momento en que las variables 
referidas al estatus social (las nueve anteriores) alcanzan puntuaciones extremadamente bajas: sin embargo, no lo explica en su totalidad, al contrario de lo que ocurría en 1991; en esta ocasión el agrupamiento deja fuera al grueso de las variables que son representativas de ciclos familiares jóvenes, conformado por la relación de fecundidad y el porcentaje de cabezas de familia menores de 35 años; de cualquier forma, las puntuaciones que ambas variables alcanzan en este segundo factor tienen signo negativo, y el valor de la relación de fecundidad es indicativo de una relación con el factor moderada. Por todo ello, este segundo factor podría asimilarse con lo que hemos dado en denominar ciclo familiar maduro. Puntuaciones positivas indicarán ciclos familiares maduros, y negativas, jóvenes.

Son las dos variables restantes, las personas principales menores de 35 años y relación de fecundidad, las que aportan el significado al tercer factor, ambas con signo positivo; en este sentido, es preciso indicar que las 14 variables restantes, esto es, no sólo las relacionadas con el estatus social, sino también las cuatro en relación con el ciclo familiar maduro, obtienen en este factor puntuaciones muy bajas. En consecuencia, estimamos que este factor hace referencia a hogares muy jóvenes, en el sentido de que los grupos de población que están en la base del cálculo de las variables de partida son los de menor edad de cada grupo: las personas principales de menor edad, la población infantil de menor edad -menor de 5 años- y las mujeres de menor edad -de entre 15 y 44 años-. Además, y como en su momento veremos, las secciones donde este factor alcanza sus mayores puntuaciones son aquellas en las que la mancha urbana se ha expandido de forma más notable, con viviendas ocupadas, en el último decenio, por lo que entendemos que este factor podría identificarse con el ciclo familiar joven. La obtención en él de puntuaciones elevadas y positivas estarían indicando que la población encuadrada en la sección correspondiente estaría atravesando las primeras fases del ciclo familiar, caracterizadas por niveles elevados de fecundidad y cabezas de familias jóvenes, al tiempo que puntuaciones elevadas y negativas estarían indicando la situación opuesta 5 .

Resulta, pues, que en el municipio de Marbella en 2001 son tres los factores que están en la base de la segregación espacial de la población. El más importante continúa siendo, igual que en 1991, el relacionado con el estatus

5. Resulta interesante indicar que en un estudio realizado sobre la ciudad de Málaga el análisis factorial indicaba la existencia de un factor basado en pocas variables y con escaso poder de explicación, pero que se identificaba de la misma manera que en nuestro caso, alcanzando puntuaciones positivas y elevadas en las entonces zonas de expansión reciente de la ciudad, tanto hacia el espacio externo de la misma, como hacia el interior, siempre sobre tejido urbano recientemente ocupado. Cfr. OCAÑA OCAÑA, M. C., Estructuras sociodemográficas y áreas sociales en la ciudad de Málaga, Sevilla 1988. 
social, factor en el que quedan embutidas las variables relacionadas con el origen de la población. En consecuencia, las diferencias espaciales en la localización de la población foránea en el municipio son consecuencias no de diferencias étnicas o culturales, sino de diferencias socioeconómicas. Por su parte, los otros dos factores muestran las dos vertientes de un mismo elemento, el ciclo familiar. Uno de ellos, el segundo, agrupa a las variables que podrían relacionarse con ciclos familiares maduros, mientras que el tercero, marginal con respecto al peso de los otros dos, nos está indicando la presencia de familias recién formadas.

Una vez identificados los factores, podemos recurrir a la hora de enriquecer el análisis a cartografiar las puntuaciones que las diferentes secciones censales alcanzan en cada una de las tres nuevas supervariables.

La distribución espacial de las puntuaciones del factor 1, que hemos identificado con el estatus social y que se muestran en la tabla 3, muestra la existencia de áreas netamente diferenciadas entre sí (figura 2). Así, aparece clara la existencia de un área de muy elevado estatus social en la porción occidental del casco marbellí, compuesta por las tres secciones costeras que se extienden al oeste del Arroyo de Las Represas (secciones 21 a 23); junto a ellas, aunque con unas puntuaciones algo más bajas podrían encuadrarse las secciones 17 y 20, colindantes por el norte con las anteriores, y que en conjunto se extienden por la mitad oeste del casco de la ciudad con mayor nivel de consolidación urbano. En el otro extremo de la escala, el estatus social más bajo continúa correspondiendo, igual que en 1991, a las secciones que se extienden por la porción más antigua de la barriada de la Divina Pastora (sección 6), la sección 3, y el área de la Colonia de La Esperanza (sección 10), a las que se une la número 11, donde se ubica la barriada de Santa Marta ${ }^{6}$. El resto de secciones presenta puntuaciones positivas más bajas que las cuatro precedentes, pero de la homogeneidad interna de la porción oriental del caso da cuenta el hecho de que tan sólo una sección, la número 4 -única con salida al mar-, obtiene puntuaciones negativas, en consonancia con las actuaciones residenciales de considerable mejor calidad que se han ejecutado en ella en los últimos años, localizadas en su práctica totalidad en la franja costera de la misma.

6. Una exposición más amplia del tejido urbano contenido en cada sección censal, y una descripción de la evolución tanto del casco de la ciudad de Marbella como de la barriada de San Pedro Alcántara puede consultarse en Natera, op. cit, 26-69. 
FIGURA 2

PUNTUACIONES DE LAS SECCIONES CENSALES EN EL FACTOR 1
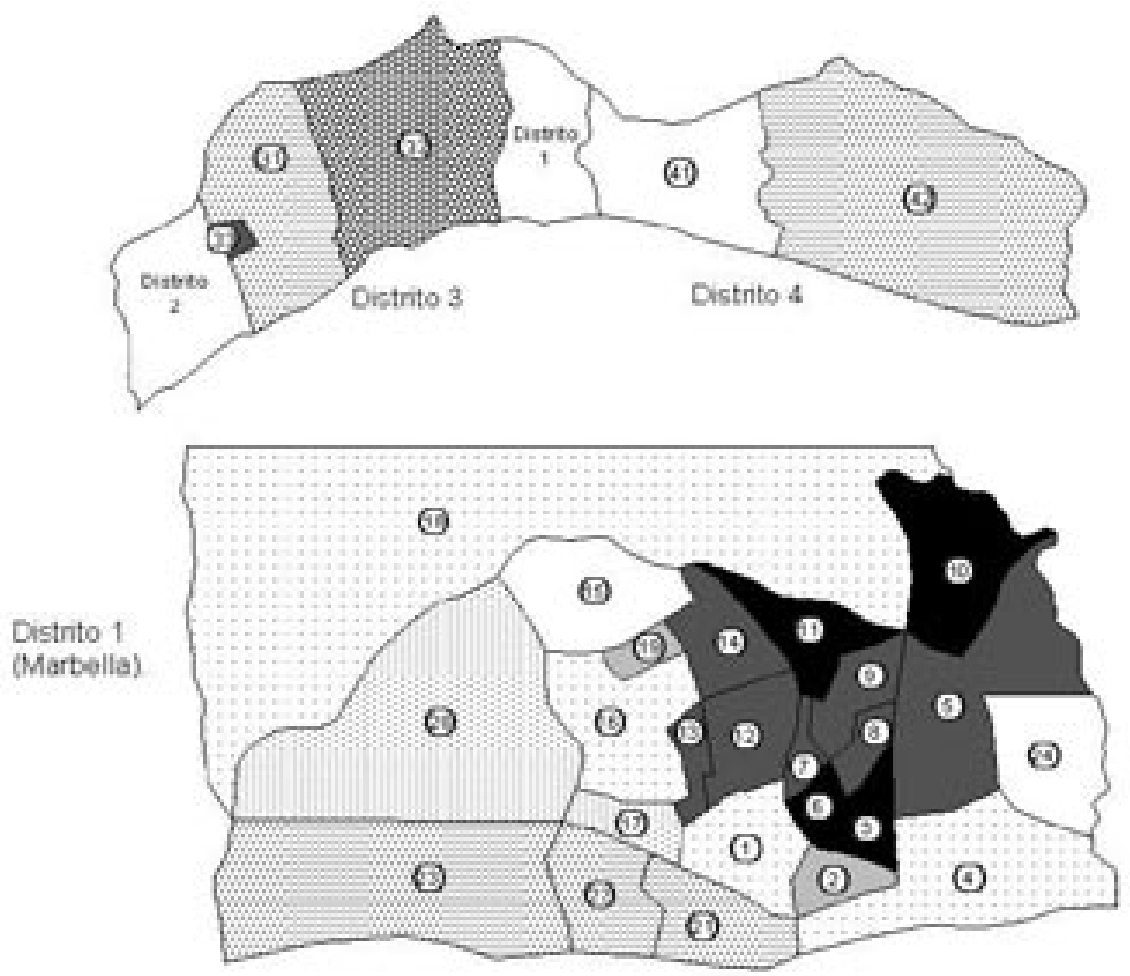

Puntuaciones:

\begin{tabular}{|r|}
\hline \\
$-2.5--2$ \\
$-2-1.5$ \\
$-1.5--1$ \\
$-1-0.5$ \\
$-0.5-0$ \\
$0-0.5$ \\
$0.5-1$ \\
$1-1.5$
\end{tabular}

Distrito 2

(Sen Fedro).

(5)

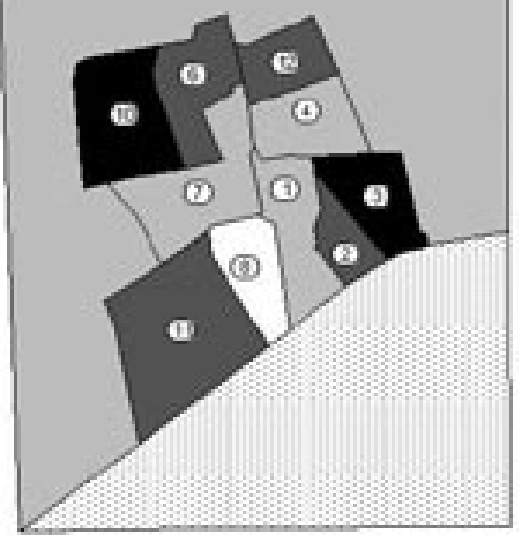


En cuanto al núcleo de San Pedro, continúa caracterizado en su totalidad por un estatus social bajo, muy bajo en el caso de las secciones de los extremos del sudeste y noroeste (zonas de Romero y de expansión más reciente del núcleo sampedreño, respectivamente), puntuando tan sólo de manera negativa -indicativo de un estatus social algo más elevado- la sección novena, que aglutina todo el espacio localizado al sur de la antigua CN-340.

Por último, es necesario indicar que son las secciones 3-2 y 4-2 las que resultan estar caracterizadas por el estatus social más elevado de todo el municipio, habiéndose registrado un descenso del mismo en estos últimos 10 años en las secciones 3-1 y 4-1, derivado de un proceso de ocupación de viviendas nuevas por parte de una población no exactamente asimilable a la preexistente, situación especialmente marcada en 4-1, asiento de parte de la expansión del casco marbellí.

Puede indicarse, por tanto, que la división entre un área de elevado estatus social y otra de bajo estatus social se mantiene diez años después en lo fundamental. La primera de ellas continua conformada por las secciones costeras occidentales del casco marbellí y las colindantes por el norte, más la mayor parte de los distritos 3 y 4; por su parte, la porción oriental del caso de la ciudad de Marbella, toda la barriada de San Pedro Alcántara y la sección tercera del tercer distrito (que engloba a La Campana) conforman la segunda de las áreas. En este sentido, las mutaciones más importantes registradas se localizan en los distritos 3 y 4, que han visto cómo las secciones colindantes con los dos núcleos históricos del municipio (San Pedro y Marbella, respectivamente), han experimentado un descenso en las puntuaciones en este factor, especialmente marcado en 3-1, y para cuya explicación debe acudirse a la proliferación de grandes cantidades de vivienda dirigida a población con un menor poder adquisitivo que la preexistente, especialmente en las áreas que han acogido parte de la expansión de los dos núcleos.

TABLA 3

\section{PUNTUACIONES EN LOS FACTORES DE LAS SECCIONES CENSALES.}

\begin{tabular}{|c|c|c|c|c|c|c|c|}
\hline Sección & Factor 1 & Factor 2 & Factor 3 & Sección & Factor 1 & Factor 2 & Factor 3 \\
\hline $1-1$ & $-0,58$ & 1,99 & 0,12 & $1-22$ & $-1,65$ & 0,52 & $-0,14$ \\
\hline $1-2$ & 0,21 & 0,93 & 0,30 & $1-23$ & $-1,69$ & 0,90 & 0,45 \\
\hline $1-3$ & 1,19 & 1,54 & 0,92 & $1-24$ & $-0,33$ & $-1,23$ & 0,16 \\
\hline $1-4$ & $-0,79$ & 0,09 & $-0,31$ & $1-25$ & 1,15 & $-2,07$ & $-0,19$ \\
\hline $1-5$ & 0,72 & $-1,35$ & 1,85 & $2-1$ & 0,05 & 0,52 & $-0,30$ \\
\hline $1-6$ & 1,16 & 1,75 & 0,08 & $2-2$ & 0,74 & 0,08 & 0,40 \\
\hline $1-7$ & 0,74 & 0,97 & $-0,55$ & $2-3$ & 1,05 & $-0,15$ & $-0,10$ \\
\hline
\end{tabular}




\begin{tabular}{|c|c|c|c|c|c|c|c|}
\hline Sección & Factor 1 & Factor 2 & Factor 3 & Sección & Factor 1 & Factor 2 & Factor 3 \\
\hline $1-8$ & 0,97 & 0,91 & 0,47 & $2-4$ & 0,25 & $-1,44$ & $-0,21$ \\
\hline $1-9$ & 0,54 & $-0,74$ & $-1,59$ & $2-5$ & 0,11 & $-1,76$ & $-0,32$ \\
\hline $1-10$ & 1,37 & 0,24 & 1,94 & $2-6$ & 0,97 & $-0,26$ & $-0,64$ \\
\hline $1-11$ & 1,00 & 0,10 & 0,11 & $2-7$ & 0,06 & 0,15 & $-1,65$ \\
\hline $1-12$ & 0,55 & 0,67 & $-0,29$ & $2-8$ & $-0,05$ & $-0,25$ & $-2,29$ \\
\hline $1-13$ & 0,64 & 0,52 & 0,74 & $2-9$ & $-1,19$ & $-0,43$ & $-0,56$ \\
\hline $1-14$ & 0,87 & 0,83 & 0,17 & $2-10$ & 1,12 & $-0,84$ & 0,03 \\
\hline $1-15$ & $-0,14$ & $-1,08$ & $-1,39$ & $2-11$ & 0,52 & $-0,38$ & $-1,44$ \\
\hline $1-16$ & $-0,66$ & 0,48 & $-0,57$ & $2-12$ & 0,91 & 0,55 & $-0,20$ \\
\hline $1-17$ & $-1,18$ & 1,69 & $-0,25$ & $3-1$ & $-1,53$ & $-0,27$ & 0,99 \\
\hline $1-18$ & $-0,85$ & $-1,88$ & $-0,50$ & $3-2$ & $-2,34$ & $-0,32$ & 0,78 \\
\hline $1-19$ & 0,08 & 0,96 & $-0,74$ & $3-3$ & 0,54 & $-0,71$ & 0,85 \\
\hline $1-20$ & $-1,37$ & $-0,49$ & 0,14 & $4-1$ & $-0,14$ & $-1,20$ & 2,83 \\
\hline $1-21$ & $-1,51$ & 0,44 & $-0,89$ & $4-2$ & $-1,52$ & 0,02 & 1,81 \\
\hline
\end{tabular}

En cuanto a la distribución espacial de las puntuaciones correspondientes al segundo factor, el ciclo familiar maduro, continua apareciendo con claridad la estrecha relación existente entre el ciclo familiar y el proceso de expansión de la ciudad (figura 3). Así, es el casco histórico del núcleo marbellí (sección 1) más la trama urbana correspondiente a las primeras actuaciones de la Divina Pastora (sección 6), las antiguas barriadas de San Bernabé y González Badía (sección 3), y la sección 17, que se extiende por la porción del tejido urbano situado inmediatamente al norte de la Avenida Ricardo Soriano, la porción del municipio que se encuentra en los ciclos familiares más maduros. A partir de aquí, y conforme nos dirigimos hacia la periferia del espacio construido, las puntuaciones van descendiendo progresivamente, en una suerte de estructura concéntrica articulada a partir de las secciones anteriores. Y, tal y como ocurría también en 1991, tanto el casco urbano de San Pedro Alcántara (con la excepción del casco fundacional, sección 1 , y el área de Los Catalanes, sección 12), como los distritos 3 y 4 vienen caracterizados por ciclos familiares menos maduros, como corresponde a su condición de áreas de asentamiento de la población más recientes que la ciudad de Marbella.

Por último, son las secciones correspondientes a las ampliaciones con viviendas principales más notables del casco urbano de Marbella (secciones 5 y 10), la sección 4-1, que ha recogido parte de este crecimiento, y 4-2, las que se sitúan en el extremo de lo que podemos considerar como ciclo familiar joven, con la práctica totalidad del distrito 2, y el grueso del núcleo de Marbella en la situación opuesta (figura 4). 
FIGURA 3

PUNTUACIONES DE LAS SECCIONES CENSALES

EN EL FACTOR 2
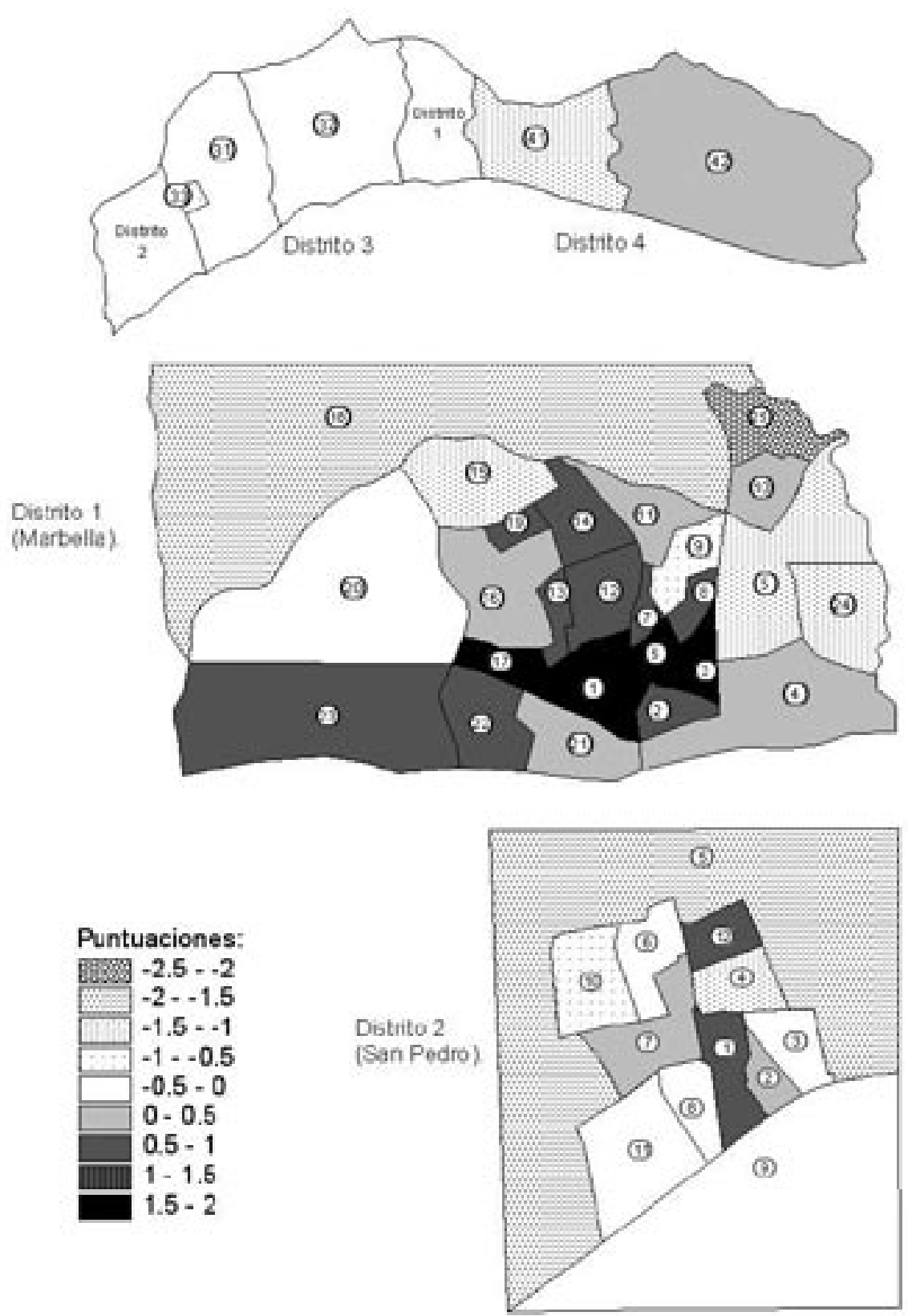
FIGURA 4

PUNTUACIONES DE LAS SECCIONES CENSALES

EN EL FACTOR 3
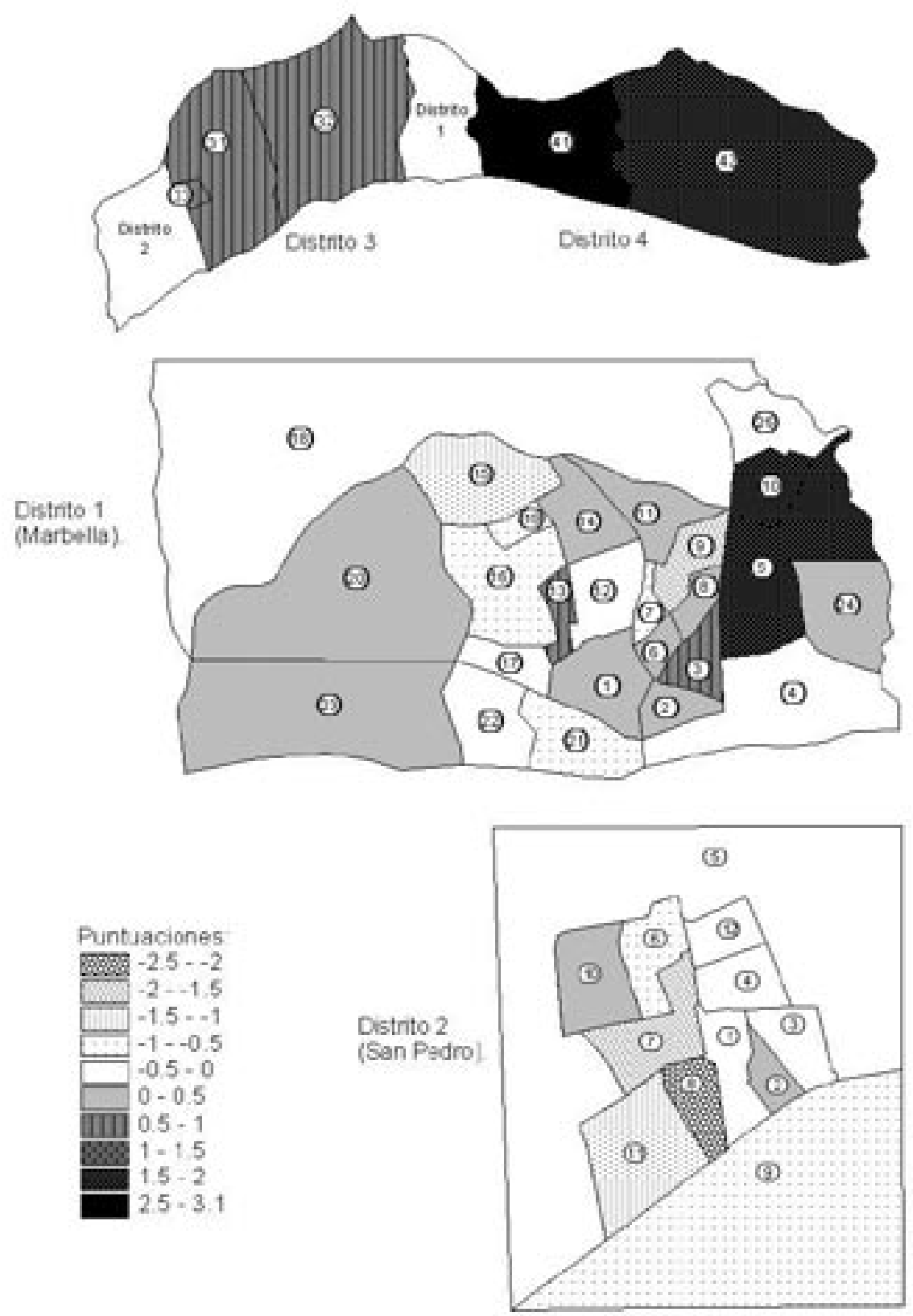


\section{CONCLUSIONES}

En las páginas anteriores hemos comprobado cómo, pese a los cambios que la población censada en Marbella ha experimentado entre 1991 y 2001, y que van más allá del mero incremento y redistribución interna, afectando a su estructura y a determinadas variables económicas y de instrucción, las dimensiones que subyacen en su distribución espacial no han variado demasiado. A través de la aplicación de un análisis factorial a las mismas 16 variables del Censo actual que fueron empleadas en otro momento, pero referidas a información de 1991, hemos comprobado cómo la distribución espacial continúa gobernada básicamente por el estatus social, y, secundariamente, por el estatus familiar. No obstante, en la actualidad, y a diferencia de lo que ocurría hace 10 años, el ciclo familiar ha quedado dividido en dos dimensiones; una la que mayor poder explicativo tiene, que hemos identificado con el ciclo familiar maduro, la segunda, de considerable menor importancia, que vendría a equivaler al ciclo familiar joven. Sin embargo, la adscripción de las diferentes secciones censales a uno u otro ciclo no se diferencia demasiado de lo que encontrábamos en 1991: las áreas centrales de la ciudad de Marbella caracterizadas por ciclos familiares maduros, tendiendo a situaciones menos maduras conforme nos dirigimos hacia la periferia; por su parte, el núcleo de San Pedro Alcántara continua inmerso, en su mayor parte, en ciclos familiares jóvenes, al igual que los distritos 2 y 3 .

Por último, las áreas en las que la caracterización de ciclo familiar joven, con ese matiz de creación reciente de los hogares, es más acusada, viene a coincidir con las zonas del núcleo marbellí en las que se ha registrado un incremento más notable de la vivienda principal, a las que hay que añadir el extremo occidental del distrito 4, afectado asimismo por la expansión espacial de la mancha urbana del casco de la ciudad de Marbella. 
